\title{
LMI sufficient conditions for the consensus of linear agents with nearly-periodic resets
}

\author{
Marcos-Cesar Bragagnolo, Irinel-Constantin Morărescu, \\ Jamal Daafouz and Pierre Riedinger *
}

June 5, 2014

\begin{abstract}
The paper focuses on the problem of consensus in the framework of hybrid systems. We consider networks of scalar agents interconnected via directed graphs. Precisely, the network consists of a large number of agents belonging to several clusters. Each cluster is represented by a fixed directed strongly connected graph and almost all the time there is no link between different clusters. In each cluster there exists a specific agent called leader. At specific instants, the leaders interact via a fixed directed strongly connected graph. In this model the agents have continuous dynamics but the states of the leaders are reseted at the instants when they interact between them. In the paper, we first characterize the consensus value of this model and show it depends only on the initial condition and the interaction topologies. Next, we provide sufficient condition in Linear Matrix Inequality (LMI) form for the global uniform exponential stability of the consensus in presence of an almost periodic reset rule. The numerical implementation of this LMI condition requires a polytopic embedding provided before illustrating the results using an academic example.
\end{abstract}

\section{Introduction}

From biology, ecology and sociology to communication and power systems, diverse areas of science and engineering deal with complex interconnected systems called networks $[24,8,12,6,22,21]$. Throughout this paper, whatever is the domain of application, the constitutive elements of the network will be called agents and their number will define the network dimension. In many fields of application, the encountered networks have often large dimension $[4,3,7]$ and they are obtained interconnecting several networks of smaller dimension. In other words, inside large networks we can detect groups of agents called clusters or communities. The agents belonging to a community are better connected $[17,16,14]$ and they agree/synchronize faster on some quantity of interest called state.

\footnotetext{
${ }^{*}$ The authors are with Université de Lorraine, CRAN, UMR 7039, 2 Avenue de la Forêt de Haye, France and CNRS, CRAN, UMR 7039. \{marcos-cesar.bragagnolo, constantin.morarescu,jamal.daafouz, pierre.riedinger $\}$ at univ-lorraine.fr
} 
The networks are often represented by graphs in which the vertices are the agents and the links define the interconnections between agents. The problem of agreement of dynamic agents belonging to a network has been studied in many frameworks depending on the application. The variations include: whether the edge set of the graph remains fixed or changes over time; whether the graph is undirected or directed; whether the dynamics is continuous or discrete; whether the state of each agent is scalar or vectorial; whether the interconnections are affected by delays or not; and whether all nodes update their states in the same time or not $[10,19,23,13,18,15]$. The agreement speed in different frameworks has also been quantified (see for instance [26, 20]). Some works have been oriented towards networks in which the global agreement cannot be reached and only local ones are obtained $[14,25]$. Some others studied the agreement in networks of agents with discrete dynamics in which any agent is linked with no more than one neighbor at each time $[5,2,11,1]$.

In this work, motivated by some behaviors encountered in social networks we address a consensus problem involving a mix of continuous and discrete dynamics. The network consists of a large number of agents belonging to several clusters. Each cluster is represented by a fixed directed strongly connected graph and almost all the time there is no link between different clusters. In each cluster there exists a specific agent called leader. At specific instants, that will be defined in the next section, the leaders interact via a fixed directed strongly connected graph. We consider a model in which the agents have continuous dynamics but the states of the leaders are reseted at some specific instants corresponding to the moments when the leaders interact between them. This model can be interpreted in terms of opinion dynamics. Each agent has an opinion that continuously evolves towards a local agreement representing the opinion of the community in which it lies. At specific instants, the leaders of the communities interact and they reset their opinion taking into account the ones of other leaders. The new opinions of the leaders will reset the values of the local agreements in each community. Iterating this process all the opinions will tend to a common value that depends only on the initial conditions and the network topology.

The rest of the paper is organized as follows. In Section 2 we formulate the problem under consideration. The agreement behavior and the possible consensus value are studied in Section 3. Sufficient conditions for the global uniform exponential stability of the consensus are provided in Section 4. This condition is given in the form of a parametric LMI. In the next section we consider the problem of numerical implementation of the proposed conditions. Precisely we have shown that only a finite number of LMIs has to be solved. Section 6 is dedicated to numerical simulation which illustrate the results. Some conclusions and perspectives are presented at the end of the paper.

Notation. The following standard notation will be used throughout the paper. The sets of nonnegative integers, real and nonnegative real numbers are denoted by $\mathbb{N}, \mathbb{R}$ and $\mathbb{R}_{+}$, respectively. For a vector $x$ we denote by $\|x\|$ its Euclidian norm. The transpose of a matrix $A$ is denoted by $A^{\top}$. Given a symmetric matrix $A \in \mathbb{R}^{n \times n}$, notation $A>0(A \geq 0)$ means that $A$ is positive (semi-)definite. By $I_{k}$ we denote the $k \times k$ identity matrix. $\mathbf{1}_{k}$ and $\mathbf{0}_{k}$ are the column vectors of size $k$ having all the components equal 1 and 0 , respectively. We also use $x\left(t_{k}^{-}\right)=\lim _{t \rightarrow t_{k}, t \leq t_{k}} x(t)$. 


\section{Problem formulation}

We consider a network of $n$ agents described by the digraph (i.e. directed graph) $\mathcal{G}=$ $(\mathcal{V}, \mathcal{E})$ where the vertex set $\mathcal{V}$ represents the set of agents and the edge set $\mathcal{E} \subset \mathcal{V} \times \mathcal{V}$ represents the interactions.

Definition $1 A$ path in a given digraph $\mathcal{G}=(\mathcal{V}, \mathcal{E})$ is a union of directed edges $\bigcup_{k=1}^{p}\left(i_{k}, j_{k}\right)$ such that $i_{k+1}=j_{k}, \forall k \in\{1, \ldots, p-1\}$.

Two nodes $i, j$ are connected in a digraph $\mathcal{G}=(\mathcal{V}, \mathcal{E})$ if there exists at least a path in $\mathcal{G}$ joining $i$ and $j$ (i.e. $i_{1}=i$ and $j_{p}=j$ ).

A strongly connected digraph $i$ s such that any two distinct nodes are connected. A strongly connected component of a digraph is a maximal subset such that any of its two distinct nodes are connected.

In the sequel, we consider that the agent set $\mathcal{V}$ is partitioned in $m$ strongly connected clusters/communities $\mathcal{C}_{1}, \ldots, \mathcal{C}_{m}$ and no link between agents belonging to different communities exists. Each community possesses one particular agent called leader and denoted in the following by $l_{i} \in \mathcal{C}_{i}, \forall i \in\{1, \ldots, m\}$. The set of leaders will be referred to as $\mathcal{L}=\left\{l_{1}, \ldots, l_{m}\right\}$. At specific time instants $t_{k}, k \geq 1$, called reset times, the leaders interact between them following a predefined interaction map $\mathcal{E}_{l} \subset \mathcal{L} \times \mathcal{L}$. We also suppose that $\mathcal{G}_{l}=\left(\mathcal{L}, \mathcal{E}_{l}\right)$ is strongly connected. The rest of the agents will be called followers and denoted by $f_{j}$. For the sake of clarity we consider that the leader is the first element of its community:

$$
\mathcal{C}_{i}=\left\{l_{i}, f_{m_{i-1}+2}, \ldots, f_{m_{i}}\right\}, \forall i \in\{1, \ldots, m\}
$$

where $m_{0}=0, m_{m}=n$ and the cardinality of $\mathcal{C}_{i}$ is given by

$$
\left|\mathcal{C}_{i}\right| \triangleq n_{i}=m_{i}-m_{i-1}, \forall i \geq 1 .
$$

In order to keep the presentation simple and making an abuse of notation, each agent will have a scalar state denoted also by $l_{i}$ for the leader $l_{i}$ and $f_{j}$ for the follower $f_{j}$. We also introduce the vectors $x=\left(l_{1}, f_{2}, \ldots, f_{m_{1}}, \ldots, l_{m}, \ldots, f_{m_{m}}\right)^{\top} \in \mathbb{R}^{n}$ and $x_{l}=\left(l_{1}, l_{2}, \ldots, l_{m}\right)^{\top} \in \mathbb{R}^{m}$ collecting all the states of the agents and all the leaders' states, respectively.

We are ready now to introduce the linear reset system describing the overall network dynamics:

$$
\begin{cases}\dot{x}(t)=-L x(t), & \forall t \in \mathbb{R}_{+} \backslash \mathcal{T} \\ x_{l}\left(t_{k}\right)=P_{l} x_{l}\left(t_{k}^{-}\right) & \forall t_{k} \in \mathcal{T} \\ x(0)=x_{0} & \end{cases}
$$

where $\mathcal{T}=\left\{t_{k} \in \mathbb{R}_{+} \mid t_{k}<t_{k+1}, \forall k \in \mathbb{N}, t_{k}\right.$ reset time $\}, L \in \mathbb{R}^{n \times n}$ is a generalized Laplacian matrix associated to the graph $\mathcal{G}$ and $P_{l} \in \mathbb{R}^{m \times m}$ is a Perron matrix associated to the graph $\mathcal{G}_{l}=\left(\mathcal{L}, \mathcal{E}_{l}\right)$. Precisely, the entries of $L$ and $P_{l}$ satisfies the following relations:

$$
\left\{\begin{array}{l}
L_{(i, j)}=0, \text { if }(i, j) \notin \mathcal{E} \\
L_{(i, j)}<0, \text { if }(i, j) \in \mathcal{E}, i \neq j \\
L_{(i, i)}=-\sum_{j \neq i} L_{i, j}, \forall i=1, \ldots, n
\end{array},\right.
$$




$$
\left\{\begin{array}{l}
P_{l(i, j)}=0, \text { if }(i, j) \notin \mathcal{E}_{l} \\
P_{l(i, j)}>0, \text { if }(i, j) \in \mathcal{E}_{l}, i \neq j \\
\sum_{j=1}^{m} P_{l(i, j)}=1, \forall i=1, \ldots, m
\end{array} .\right.
$$

The values $L_{(i, j)}$ and $P_{l(i, j)}$ represent the weight of the state of the agent $j$ in the updating process of the state of agent $i$ when using the continuous and discrete dynamics, respectively. These values describe the level of "democracy" inside each community and in the leaders' network.

In particular, $L$ has the following block diagonal structure

$$
L=\left(\begin{array}{ccc}
L_{1} & & \\
& \ddots & \\
& & L_{m}
\end{array}\right), L_{i} \in \mathbb{R}^{n_{i}}
$$

with $L_{i} \mathbf{1}_{n_{i}}=\mathbf{0}_{n_{i}}$ and $P_{l} \mathbf{1}_{m}=\mathbf{1}_{m}$. Due to the strong connectivity of $\mathcal{C}_{i}, i=1, m$ and $\mathcal{G}_{l}, 0$ is simple eigenvalue of each $L_{i}$ and 1 is simple eigenvalue of $P_{l}$.

This model can be interpreted in terms of opinion dynamics or decision making process in social networks. The network is partitioned in a number of communities. The opinion of each agent continuously evolves taking into account the opinion of its neighbors. At the time $t_{k}$ the leaders interact in a dedicated network and reset their opinion. We show that, if no external perturbation occurs and the process continue for a long period of time, all the opinions will converge towards a common one. This global agreement opinion depends on the level of "democracy" inside each community and on the level of "democracy" in the leaders' network.

\section{Agreement behavior}

In this section we assume that system (2) achieves consensus and we characterize the possible values for it. Firstly, we show that each agent tracks a local agreement function which is piecewise constant. In the second subsection we prove that the vector of local agreements lies in a subspace defined by the system's dynamics and initial condition. Therefore, if the consensus is achieved and the corresponding consensus value is $x^{*}$ than $x^{*} \mathbf{1}_{m}$ belongs to the same subspace. Moreover, this value is determined only by the initial condition of the network and by the interconnection structure.

As we have noticed $\mathbf{1}_{n_{i}}$ is the right eigenvector of $L_{i}$ associated with the the eigenvalue 0 and $\mathbf{1}_{m}$ is the right eigenvector of $P_{l}$ associated with the eigenvalue 1 . In the sequel, we denote by $w_{i}$ the left eigenvector of $L_{i}$ associated with the eigenvalue 0 such that $w_{i}^{\top} \mathbf{1}_{n_{i}}=1$. Similarly, let $v=\left(v_{1}, \ldots, v_{m}\right)^{\top}$ be the left eigenvector of $P_{l}$ associated with the eigenvalue 1 such that $v^{\top} \mathbf{1}_{m}=1$. Due to the structure (1) of the communities, we emphasize that each vector $w_{i}$ can be decomposed in its first component $w_{i, l}$ and the rest of its components grouped in the vector $w_{i, f}$. 


\subsection{Local agreements}

Let us first recall a well known result concerning the consensus in networks of agents with continuous time dynamics (see [19] for instance).

Lemma 2 Let $\mathcal{G}$ be a strongly connected digraph and $L$ the corresponding Laplacian matrix. Consider a network of agents whose collective dynamics is described by $\dot{x}(t)=-L x(t)$. Let us also consider $L \mathbf{1}=\mathbf{0}, u^{\top} L=\mathbf{0}$ and $u^{\top} \mathbf{1}=1$. Then, the agents asymptotically reach a consensus and the consensus value is given by $x^{*}=u^{\top} x(0)$. Moreover, the vector $u$ defines an invariant subspace for the collective dynamics: $u^{\top} x(t)=u^{\top} x(0), \forall t \geq 0$

Remark 1 When dynamics (2) is considered, Lemma 2 implies that between two reset instants $t_{k}$ and $t_{k+1}$, the agents belonging to the same community converge to a local agreement defined by $x_{i}^{*}(k)=w_{i}^{\top} x_{\mathcal{C}_{i}}\left(t_{k}\right)$ where $x_{\mathcal{C}_{i}}(\cdot)$ is the vector collecting the states of the agents belonging to the cluster $\mathcal{C}_{i}$. Nevertheless, at the reset times the value of the local agreement can change. Thus,

$$
\begin{aligned}
& w_{i}^{\top} x(t)=w_{i}^{\top} x_{\mathcal{C}_{i}}\left(t_{k}\right), \forall t \in\left(t_{k}, t_{k+1}\right) \text { and possibly } \\
& w_{i}^{\top} x_{\mathcal{C}_{i}}(t) \neq w_{i}^{\top} x_{\mathcal{C}_{i}}\left(t_{k}\right), \text { for } t \notin\left(t_{k}, t_{k+1}\right)
\end{aligned}
$$

Therefore, the agents whose collective dynamics is described by the hybrid system (2), may reach a consensus only if the local agreements converge one to each other.

\subsection{Consensus value}

Before presenting our next result, let us introduce the following vectors:

$$
\begin{aligned}
& x^{*}(t)=\left(x_{1}^{*}(t), x_{2}^{*}(t), \ldots, x_{m}^{*}(t)\right)^{\top} \in \mathbb{R}^{m} \\
& u=\left(v_{1} / w_{1, l}, v_{2} / w_{2, l}, \ldots, v_{m} / w_{m, l}\right)^{\top} \in \mathbb{R}^{m}
\end{aligned}
$$

It is noteworthy that $x^{*}(t)$ is time-varying but piecewise constant: $x^{*}(t)=x^{*}(k) \forall t \in$ $\left(t_{k}, t_{k+1}\right)$.

Proposition 3 Consider the system (2) with $L$ and $P_{l}$ defined by (3) and (4), respectively. Then,

$$
u^{\top} x^{*}(t)=u^{\top} x^{*}(0), \forall t \in \mathbb{R}_{+} .
$$

Proof: Let us introduce the matrix of the left eigenvectors of the communities:

$$
W=\left[\begin{array}{cccc}
w_{1}^{\top} & 0 & \cdots & 0 \\
0 & w_{2}^{\top} & \cdots & 0 \\
\vdots & \vdots & \ddots & \vdots \\
0 & 0 & \cdots & w_{m}^{\top}
\end{array}\right] \in \mathbb{R}^{m \times n}
$$

Then, the following relation holds:

$$
x^{*}(t)=W x(t) \quad \forall t \in \mathbb{R}_{+} \backslash \mathcal{T}
$$


Since $w_{i}=\left(w_{i l}, w_{i f}\right)$, we define a permutation matrix $T$ such that $W T^{\top}=U=$ $\left(U_{1}, U_{2}\right)$. The matrix $U_{1}$ is a diagonal matrix corresponding to the leaders' components $w_{i, l}$, while $U_{2}$ is a block diagonal matrix corresponding to the followers' components $w_{i, f}$. In other terms

$$
\begin{gathered}
U_{1}=\left[\begin{array}{cccc}
w_{1, l} & 0 & \cdots & 0 \\
0 & w_{2, l} & \cdots & 0 \\
\vdots & \vdots & \ddots & \vdots \\
0 & 0 & \cdots & w_{m, l}
\end{array}\right] \in \mathbb{R}^{m \times m} \\
U_{2}=\left[\begin{array}{cccc}
w_{1, f} & 0 & \cdots & 0 \\
0 & w_{2, f} & \cdots & 0 \\
\vdots & \vdots & \ddots & \vdots \\
0 & 0 & \cdots & w_{m, f}
\end{array}\right] \in \mathbb{R}^{m \times(n-m)} .
\end{gathered}
$$

Finally, we can rewrite equation (9) as:

$$
x^{*}(t)=W T^{\top} x(t)=U \cdot\left(x_{l}(t), x_{f}(t)\right) .
$$

Note that at the reset time $t_{k}$ the vector $x_{f}$ remains the same, i.e. $x_{f}\left(t_{k}\right)=x_{f}\left(t_{k}^{-}\right)$ . This yields

$$
\begin{aligned}
x^{*}\left(t_{k}\right)-x^{*}\left(t_{k}^{-}\right) & =U \cdot\left(x_{l}\left(t_{k}\right)-x_{l}\left(t_{k}^{-}\right), x_{f}\left(t_{k}\right)-x_{f}\left(t_{k}^{-}\right)\right) \\
& =U \cdot\left(x_{l}\left(t_{k}\right)-x_{l}\left(t_{k}^{-}\right), 0\right) \\
& =U_{1} \cdot\left(x_{l}\left(t_{k}\right)-x_{l}\left(t_{k}^{-}\right)\right)+U_{2} \cdot 0 \\
& =U_{1}\left(x_{l}\left(t_{k}\right)-x_{l}\left(t_{k}^{-}\right)\right) .
\end{aligned}
$$

Thus,

$$
\left.x^{*}\left(t_{k}\right)=x^{*}\left(t_{k}^{-}\right)+U_{1} \cdot\left(P_{l}-I_{m}\right) x_{l}\left(t_{k}^{-}\right)\right) .
$$

Multiplying equation (13) by $u^{\top}$ and using $u^{\top} U_{1}=v^{\top}$ one obtains

$$
\begin{aligned}
u^{\top} x^{*}\left(t_{k}^{+}\right) & =u^{\top} x^{*}\left(t_{k}^{-}\right)+u^{\top} U_{1}\left(P_{l}-I_{m}\right) x_{l} \\
& =u^{\top} x^{*}\left(t_{k}^{-}\right)+v^{\top}\left(P_{l}-I_{m}\right) \\
& =u^{\top} x^{*}\left(t_{k}^{-}\right)+v^{\top}-v^{\top} \\
& =u^{\top} x^{*}\left(t_{k}^{-}\right)
\end{aligned}
$$

According to Remark $1, x^{*}(t)$ remains constant for all $t \in\left(t_{k}, t_{k+1}\right)$ leading to

$$
u^{\top} x^{*}(t)=u^{\top} x^{*}(0) \quad \forall t \in \mathbb{R}_{+} .
$$

Corollary 1 Consider the system (2) with $L$ and $P_{l}$ defined by (3) and (4), respectively. Assuming the agents of this system reach a consensus, the consensus value is

$$
x^{*}=\frac{u^{\top} W x(0)}{\sum_{i=1}^{m} u_{i}} .
$$


Proof: Let $x^{*}$ be the consensus value reached by the system (2). It means that $x(t) \rightarrow$ $x^{*} \mathbf{1}_{n}$. Thus, when $t$ goes to $\infty$ in (15) one obtains

$$
u^{\top} x^{*} \mathbf{1}_{n}=u^{\top} x^{*}(0)=u^{\top} W x(0)
$$

leading to (16).

In order to simplify the presentation and without loss of generality, in what follows, we consider that $\sum_{i=1}^{m} u_{i}=1$.

Remark 2 It is important to note that the consensus value depends only on the system matrices $L, P_{l}$ and does not depend on the reset sequence $\mathcal{T}$. This can be interpreted as: the consensus depends only on the weight of the agents inside communities and on the wight of leaders in the leaders' network. Thus the level of "democracy" inside communities and in the leaders' network are the only factors influencing the consensus value.

A trivial result which may be seen as a consequence of Corollary 1 is the following.

Corollary 2 If the matrices $L, P_{l}$ are symmetric (i.e. $i^{\text {th }}$ agent takes into account the state of $j^{\text {th }}$ agent as far as $j^{\text {th }}$ takes into account the $i^{\text {th }}$ one and they give the same importance one to another) the consensus value is the average of the initial states.

Proof: In this case $w_{i}=\frac{1}{n_{i}} \mathbf{1}_{n_{i}}$ and $v=\frac{1}{m} \mathbf{1}_{m}$ which leads to $u=\left(\frac{n_{1}}{m}, \frac{n_{2}}{m}, \ldots, \frac{n_{m}}{m}\right)$. The result follows from (16).

\section{Stability analysis}

The stability analysis of the equilibrium point $x^{*}$ will be given by means of some LMI conditions. Precisely, we recall and adapt some results presented in [9]. Since the consensus value is computed in the previous section we can first define the disagreement vector $y=x-x^{*} \mathbf{1}_{n}$. We also introduce an extended stochastic matrix $P_{e x}$ as follows:

$$
P_{e x}=T^{\top}\left[\begin{array}{cc}
P_{l} & 0 \\
0 & I_{n-m}
\end{array}\right] T
$$

where $T$ is the permutation matrix used in the proof of Proposition 3. It is noteworthy that $L \mathbf{1}_{n}=\mathbf{0}_{n}$ and $P_{e x} \mathbf{1}_{n}=\mathbf{1}_{n}$. Thus, the disagreement dynamics is exactly the same as the system one:

$$
\left\{\begin{array}{ll}
\dot{y}(t)=-L y(t), & \forall t \in \mathbb{R}_{+} \backslash \mathcal{T} \\
y\left(t_{k}\right)=P_{\text {ex }} y\left(t_{k}^{-}\right) & \forall t_{k} \in \mathcal{T} \\
y(0)=y_{0} &
\end{array} .\right.
$$

Now we have to analyze the stability of the equilibrium point $y^{*}=\mathbf{0}_{n}$ for the system (18). We note that Theorem 2 in [9] cannot be directly applied due to the marginal stability of the matrices $L$ and $P_{e x}$. 
The reset sequence is defined such that $t_{k+1}-t_{k}=\delta+\delta^{\prime}$ where $\delta \in \mathbb{R}_{+}$is fixed and $\delta^{\prime} \in \Delta$ with $\Delta \subset \mathbb{R}_{+}$a compact set. Thus the set of reset times $\mathcal{T}$ belongs to the set of all admissible reset sequences associated with $\Delta$ :

$$
\Phi(\Delta) \triangleq\left\{\left\{t_{k}\right\}_{k \in \mathbb{N}}, t_{k+1}-t_{k}=\delta+\delta_{k}^{\prime}, \delta_{k}^{\prime} \in \Delta, \forall k \in \mathbb{N}\right\}
$$

where we always consider $t_{0}=0$.

We recall that for any $\mathcal{T} \in \Phi(\Delta)$ and any initial condition $x_{0}$ the system (2) has a unique solution denoted by $\varphi_{\mathcal{T}}\left(t, x_{0}\right)$.

Definition 4 We say that the equilibrium $y^{*}=\mathbf{0}_{n}$ of the system (18) is Globally Uniformly Exponentially Stable (GUES) with respect to the set of reset sequences $\Phi(\Delta)$ if there exist positive scalars $c, \lambda$ such that for any $\mathcal{T} \in \Phi(\Delta)$, any $y_{0} \in \mathbb{R}^{n}$, and any $t \geq 0$

$$
\left\|\varphi\left(t, y_{0}\right)\right\| \leq c e^{-\lambda t}\left\|y_{0}\right\|
$$

The following theorem is instrumental:

Theorem 5 (Theorem 1 in [9]) Consider the system (18) with the set of reset times $\mathcal{T} \in \Phi(\Delta)$. The equilibrium $y^{*}=\mathbf{0}_{n}$ is GUES if and only if there exists a positive function $V: \mathbb{R}^{n} \mapsto \mathbb{R}_{+}$strictly convex,

$$
V(y)=y^{\top} S_{[y]} y,
$$

homogeneous (of second order), $S_{[\cdot]}: \mathbb{R}^{n} \mapsto \mathbb{R}^{n \times n}, S_{[y]}=S_{[y]}^{\top}=S_{[a y]}>0, \forall x \neq$ $0, a \in \mathbb{R}, a \neq 0, V(0)=0$, such that $V\left(y\left(t_{k}\right)\right)>V\left(y\left(t_{k+1}\right)\right)$ for all $y\left(t_{k}\right) \neq 0, k \in$ $\mathbb{N}$ and any of the possible reset sequences $\mathcal{T} \in \Phi(\Delta)$.

In the sequel, we define a quasi-quadratic Lyapunov function satisfying Theorem 5 by means of some LMI. Therefore, the following result gives sufficient conditions for the stability of the equilibrium point $y^{*}=\mathbf{0}_{n}$ for the system (18) or equivalently of $x^{*} \mathbf{1}_{n}$ for the system (2).

Theorem 6 Consider the system (2) with $\mathcal{T}$ in the admissible reset sequences $\Phi(\Delta)$. If there exist matrices $S\left(\delta^{\prime}\right), S(\cdot): \Delta \mapsto \mathbb{R}^{n \times n}$ continuous with respect to $\delta^{\prime}, S\left(\delta^{\prime}\right)=$ $S^{\top}\left(\delta^{\prime}\right)>0, \delta^{\prime} \in \Delta$ such that the LMI

$$
\begin{aligned}
& \left(I_{n}-\mathbf{1}_{n} u^{\top} W\right)^{\top} S\left(\delta_{a}\right)\left(I_{n}-\mathbf{1}_{n} u^{\top} W\right)- \\
& \left(Y\left(\delta_{a}\right)-\mathbf{1}_{n} u^{\top} W\right)^{\top} S\left(\delta_{b}\right)\left(Y\left(\delta_{a}\right)-\mathbf{1}_{n} u^{\top} W\right)>0, \\
& Y\left(\delta_{a}\right) \triangleq P_{e x} e^{-L\left(\delta+\delta_{a}\right)}
\end{aligned}
$$

is satisfied on span $\left\{\mathbf{1}_{n}\right\}^{\perp}$ for all $\delta_{a}, \delta_{b} \in \Delta$, then $x^{*}$ is globally uniformly exponentially stable for (2). Moreover, the stability is characterized by the quasi-quadratic Lyapunov function $V(t)=\max _{\delta^{\prime} \in \Delta}\left(x(t)-x^{*} \mathbf{1}_{n}\right)^{\top} S\left(\delta^{\prime}\right)\left(x(t)-x^{*} \mathbf{1}_{n}\right)$ satisfying $V\left(t_{k}\right)>$ $V\left(t_{k+1}\right)$. 
Proof: Using the disagreement vector $y(t)=x(t)-x^{*} \mathbf{1}_{n}$ and supposing that there exist matrices $S\left(\delta^{\prime}\right)$ satisfying (21) for all $\delta_{a}, \delta_{b} \in \Delta$ we define the Lyapunov matrix

$$
S_{[y]}=S\left(\delta^{*}(y)\right) \text { with } \delta^{*}(y)=\arg \max _{\delta^{\prime} \in \Delta} y^{\top} S\left(\delta^{\prime}\right) y
$$

Following [9] the Lyapunov function

$$
V(y)=y^{\top} S_{[y]} y=\max _{\delta^{\prime} \in \Delta} y^{\top} S\left(\delta^{\prime}\right) y,
$$

is convex and homogeneous of the second order.

Let us show now that $S(\cdot)$ solution of (21) ensures that $V(\cdot)$ defined above satisfies Theorem 5 .

We note first that any $x\left(t_{k}\right) \in \mathbb{R}^{n}$ can be decomposed as $x\left(t_{k}\right)=\bar{x}\left(t_{k}\right)+\tilde{x}\left(t_{k}\right)$ with $\bar{x}\left(t_{k}\right) \in \operatorname{span}\left\{\mathbf{1}_{n}\right\}^{\perp}$ and $\tilde{x}\left(t_{k}\right) \in \operatorname{span}\left\{\mathbf{1}_{n}\right\}$. Moreover,

$$
\left(I_{n}-\mathbf{1}_{n} u^{\top} W\right) \tilde{x}\left(t_{k}\right)=0,\left(Y\left(\delta_{a}\right)-\mathbf{1}_{n} u^{\top} W\right) \tilde{x}\left(t_{k}\right)=0
$$

hence

$$
\begin{aligned}
& \left(I_{n}-\mathbf{1}_{n} u^{\top} W\right) x\left(t_{k}\right)=\left(I_{n}-\mathbf{1}_{n} u^{\top} W\right) \bar{x}\left(t_{k}\right), \\
& \left(Y\left(\delta_{a}\right)-\mathbf{1}_{n} u^{\top} W\right) x\left(t_{k}\right)=\left(Y\left(\delta_{a}\right)-\mathbf{1}_{n} u^{\top} W\right) \bar{x}\left(t_{k}\right)
\end{aligned}
$$

Thus, the LMI (21) yields

$$
\begin{aligned}
& x\left(t_{k}\right)^{\top}\left(I_{n}-\mathbf{1}_{n} u^{\top} W\right)^{\top} S\left(\delta_{a}\right)\left(I_{n}-\mathbf{1}_{n} u^{\top} W\right) x\left(t_{k}\right)> \\
& x\left(t_{k}\right)^{\top}\left(Y\left(\delta_{a}\right)-\mathbf{1}_{n} u^{\top} W\right)^{\top} S\left(\delta_{b}\right)\left(Y\left(\delta_{a}\right)-\mathbf{1}_{n} u^{\top} W\right) x\left(t_{k}\right), \\
& \quad \forall \delta_{a}, \delta_{b} \in \Delta
\end{aligned}
$$

Consequently, using $u^{\top} W x\left(t_{k}\right)=x^{*}$ one gets

$$
\begin{aligned}
& \left(x\left(t_{k}\right)-\mathbf{1}_{n} x^{*}\right)^{\top} S\left(\delta_{a}\right)\left(x\left(t_{k}\right)-\mathbf{1}_{n} x^{*}\right)> \\
& \left(Y\left(\delta_{a}\right) x\left(t_{k}\right)-\mathbf{1}_{n} x^{*}\right)^{\top} S\left(\delta_{b}\right)\left(Y\left(\delta_{a}\right) x\left(t_{k}\right)-\mathbf{1}_{n} x^{*}\right), \\
& \forall \delta_{a}, \delta_{b} \in \Delta, x\left(t_{k}\right) \in \mathbb{R}^{n}
\end{aligned}
$$

For any $\left\{t_{k}\right\}_{k \in \mathbb{N}} \in \Phi(\Delta)$ we have $x\left(t_{k+1}\right)=Y\left(\delta_{k}^{\prime}\right) x\left(t_{k}\right)$ with some $\delta_{k}^{\prime} \in \Delta$. Thus, for $\delta_{a}=\delta_{k}^{\prime},(23)$ rewrites as:

$$
\begin{aligned}
& \left(x\left(t_{k}\right)-x^{*} \mathbf{1}_{n}\right)^{\top} S\left(\delta_{k}^{\prime}\right)\left(x\left(t_{k}\right)-x^{*} \mathbf{1}_{n}\right)> \\
& \left(x\left(t_{k+1}\right)-x^{*} \mathbf{1}_{n}\right)^{\top} S\left(\delta_{b}\right)\left(x\left(t_{k+1}\right)-x^{*} \mathbf{1}_{n}\right) \\
& \quad \forall \delta_{k}^{\prime}, \delta_{b} \in \Delta, x\left(t_{k}\right) \in \mathbb{R}^{n}
\end{aligned}
$$


or equivalently

$$
y\left(t_{k}\right)^{\top} S\left(\delta_{k}^{\prime}\right) y\left(t_{k}\right)>y\left(t_{k+1}\right)^{\top} S\left(\delta_{b}\right) y\left(t_{k+1}\right) \quad \forall \delta_{k}^{\prime}, \delta_{b} \in \Delta
$$

Taking $\delta_{b}=\delta^{*}\left(y\left(t_{k+1}\right)\right)$, defined by (22) one obtains

$$
V\left(y\left(t_{k}\right)\right)>y\left(t_{k}\right)^{\top} S\left(\delta_{k}^{\prime}\right) y\left(t_{k}\right)>V\left(y\left(t_{k+1}\right)\right)
$$

for all $y\left(t_{k}\right)$, which ends the proof.

\section{Numerical implementation}

In order to render this paper self-contained, in this section we consider the problem of approximation of the parametric LMI (21) by a finite number of conditions using polytopic embeddings.

\subsection{Periodic resets}

If $\Delta=\{0\}$ i.e. the resets take place periodically with the period $\delta$, Theorem 6 is rephrased as follows.

Corollary 3 If there exists a positive definite matrix $S$ such that the LMI

$$
\begin{aligned}
& \left(I_{n}-\mathbf{1}_{n} u^{\top} W\right)^{\top} S\left(I_{n}-\mathbf{1}_{n} u^{\top} W\right)- \\
& \left(Y(\delta)-\mathbf{1}_{n} u^{\top} W\right)^{\top} S\left(Y(\delta)-\mathbf{1}_{n} u^{\top} W\right)>0, \\
& Y(\delta) \triangleq P_{e x} e^{-L(\delta)}
\end{aligned}
$$

is satisfied on $\operatorname{span}\left\{\mathbf{1}_{n}\right\}^{\perp}$, then $x^{*}$ is globally uniformly exponentially stable for (2). Moreover, the stability is characterized by the quadratic Lyapunov function $V(t)=$ $\left(x(t)-x^{*} \mathbf{1}_{n}\right)^{\top} S\left(x(t)-x^{*} \mathbf{1}_{n}\right)$ satisfying $V\left(t_{k}\right)>V\left(t_{k+1}\right)$.

In this case the parametric LMI (21) is simply replaced by the LMI (24) and no numerical difficulties arise.

\subsection{Nearly-periodic resets}

In this case, to replace the parametric LMI (21) by a finite number of LMIs we have to deal with the parametric uncertainty $\delta_{k}^{\prime}$ in the definition (19) of the admissible reset sequence. As in [9] the matrix exponential $e^{-L \delta_{a}}$ is approximated by its $h-$ order Taylor expansion $\sum_{i=0}^{h} \frac{(-L)^{i}}{i !} \delta_{a}^{i}$. Thus the set $\left\{X \in \mathbb{R}^{n \times n} \mid X=e^{-L \delta_{a}}, \delta_{a} \in \Delta\right\}$ can be embedded into the polytopic set defined by the vertices $Z_{1}, \ldots, Z_{h+1}$ where 


$$
\begin{aligned}
& Z_{1}=I_{n} \\
& Z_{i}=\sum_{l=0}^{i} \frac{(-L)^{l}}{l !} \delta_{\text {max }}^{l}, \forall i \in\{1, \ldots, h+1\}
\end{aligned}
$$

with $\delta_{\max }=\max _{\delta^{\prime} \in \Delta} \delta^{\prime}$. Then, Theorem 6 can be replaced by the following result.

Theorem 7 Consider the system (2) with $\mathcal{T}$ in the admissible reset sequences $\Phi(\Delta)$. If there exist symmetric positive definite matrices $S_{i}, 0 \leq i \leq h+1$ such that the LMI

$$
\begin{aligned}
& \left(I_{n}-\mathbf{1}_{n} u^{\top} W\right)^{\top} S_{i}\left(I_{n}-\mathbf{1}_{n} u^{\top} W\right)- \\
& \left(Y(\delta) Z_{i}-\mathbf{1}_{n} u^{\top} W\right)^{\top} S_{j}\left(Y(\delta) Z_{i}-\mathbf{1}_{n} u^{\top} W\right)>0, \\
& Y(\delta) \triangleq P_{e x} e^{-L(\delta)}
\end{aligned}
$$

is satisfied on $\operatorname{span}\left\{\mathbf{1}_{n}\right\}^{\perp}$ for all $i, j \in\{\mathbf{0}, \ldots, h+1\}$, then $x^{*}$ is globally uniformly exponentially stable for (2).

Proof: Assume that the set of LMIs (25) is satisfied for a set of matrices $S_{i}, 0 \leq i \leq$ $h+1$. Thus,

$$
\begin{aligned}
& \left(I_{n}-\mathbf{1}_{n} u^{\top} W\right)^{\top}\left(\sum_{i=1}^{h+1} \mu_{i} S_{i}\right)\left(I_{n}-\mathbf{1}_{n} u^{\top} W\right)- \\
& \left(Y(\delta) \sum_{i=1}^{h+1} \mu_{i} Z_{i}-\mathbf{1}_{n} u^{\top} W\right)^{\top}\left(\sum_{i=1}^{h+1} \mu_{j} S_{j}\right) \times \\
& \left(Y(\delta) \sum_{i=1}^{h+1} \mu_{i} Z_{i}-\mathbf{1}_{n} u^{\top} W\right)>0,
\end{aligned}
$$

is satisfied for all $\mu_{i}, \mu_{j} \in[0,1], i, j \in\{1, \ldots, h+1\}$ such that $\sum_{i=1}^{h+1} \mu_{i}=\sum_{j=1}^{h+1} \mu_{j}=$ 1. It is noteworthy that the polytopic embedding provided above implies that for all $\delta_{a} \in\left[0, \delta_{\max }\right]$ there exists the set of scalars $\mu_{i} \in[0,1]$ such that $e^{-L \delta_{a}}=\sum_{i=1}^{h+1} \mu_{i} Z_{i}$ and $\sum_{i=1}^{h+1} \mu_{i}=1$. In other words, Theorem 6 holds with $S\left(\delta^{\prime}\right)=\sum_{i=1}^{h+1} \mu_{i}\left(\delta^{\prime}\right) S_{i}$.

\section{Illustrative Example}

An academic example consisting in a network of 5 agents partitioned in 2 clusters $\left(n_{1}=3, n_{2}=2\right)$ is used in the sequel to illustrate the theoretical results. We consider the dynamics (2) with 


$$
L=\left[\begin{array}{ccccc}
4 & -2 & -2 & 0 & 0 \\
-1 & 1 & 0 & 0 & 0 \\
0 & -2 & 2 & 0 & 0 \\
0 & 0 & 0 & 3 & -3 \\
0 & 0 & 0 & -1 & 1
\end{array}\right], P_{l}=\left[\begin{array}{cc}
0.4500 & 0.5500 \\
0.2500 & 0.7500
\end{array}\right]
$$

and the reset sequence given by $\delta=0.5$ and $\delta_{k}^{\prime}$ randomly chosen in $\Delta=[0,0.2]$. The initial condition of the system is $x(0)=(1,4,9,2,5)$ and the corresponding consensus value computed by (16) is $x^{*}=4.1587$. The convergence of the 5 agents towards $x^{*}$ is illustrated in Figure 1 emphasizing that the leaders trajectories are non-smooth while the followers trajectories are. The jumps, convexity and decreasing of the Lyapunov function defined by Theorem 6 are pointed out in Figure 2 .

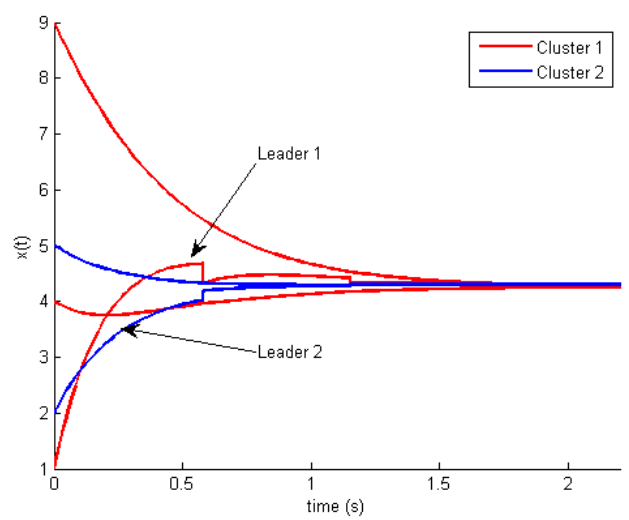

Figure 1: The state-trajectories of the agents converging to the calculated consensus value.

Larger networks have also been numerically analyzed but due to the space limitation we do not provide the simulations here.

\section{Conclusions and perspectives}

In this paper we have considered the problem of consensus for linear reset systems. The contribution of the paper is twofold: firstly, we define the consensus value for the systems under consideration and secondly, we provide their stability analysis using the Lyapunov framework. The results are easy to implement since the sufficient conditions for stability are given in LMI form and the corresponding Lyapunov function is defined by the solutions of these LMIs.

An interesting issue that will be considered in future works is the design of the reset mapping that allows to reach a prescribed consensus value. 


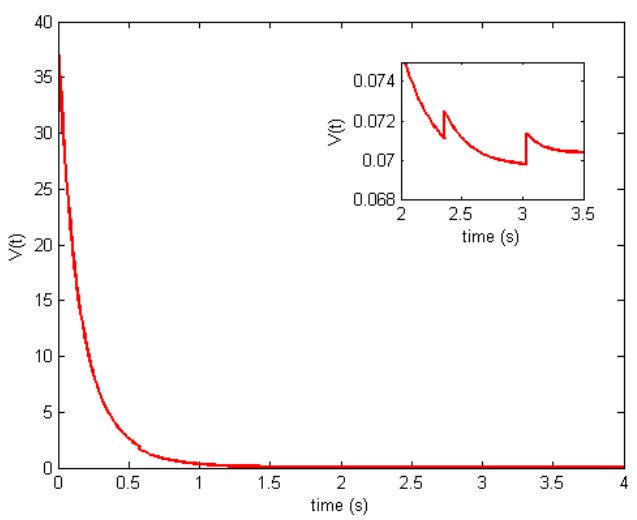

Figure 2: The behavior of the Lyapunov function given by Theorem 6.

\section{References}

[1] B.D.O. Anderson, C. Yu, and A.S. Morse. Convergence of periodic gossiping algorithms. Springer, New York, 2010.

[2] T.C. Aysal, M.E. Yildriz, A.D. Sarwate, and A. Scaglione. Broadcast gossip algorithms for consensus. IEEE Transactions on Signal Processing, 57:27482761, 2009.

[3] V.D. Blondel, J.-L. Guillaume, R. Lambiotte, and E. Lefebvre. Fast unfolding of communites in large networks. Journal of Statistical Mechanics: Theory and Experiment, P10008(10), 2008.

[4] S. Boccaletti, V. Latora, Y. Moreno, M. Chavez, and D.-U. Hwang. Complex networks: Structure and dynamics. Physics Report, 424(4-5):175-308, 2006.

[5] S. Boyd, A. Ghosh, B. Prabhakar, and D. Shah. Randomized gossip algorithms. IEEE Transactions on Information Theory, 52(6):2508-2530, 2006.

[6] D. Gfeller and P. De Los Rios. Spectral coarse graining and synchronization in oscillator networks. Phys. Rev. Lett., 100:174104, 2008.

[7] I. Hanski. Metapopulation dynamics. Nature, 396:41-49, 1998.

[8] R. Hegselmann and U. Krause. Opinion dynamics and bounded confidence models, analysis, and simulation. Journal of Artificial Societies and Social Simulation, 5(3), 2002.

[9] L. Hetel, J. Daafouz, S. Tarbouriech, and C. Prieur. Stabilization of linear impulsive systems through a nearly-periodic reset. Nonlinear Analysis: Hybrid Systems, 7:4-15, 2013. 
[10] A. Jadbabaie, J. Lin, and A. S. Morse. Coordination of groups of mobile autonomous agents using nearest neighbor rules. IEEE Trans. on Automatic Control, 48(6):988-1001, 2003.

[11] J. Liu, B.D.O. Anderson, M. Cao, and A.S. Morse. Analysis of accelerated gossip algorithms. In Proceedings IEEE Conference on Decision and Control, pages 871-876, 2009.

[12] J. Lorenz. A stabilization theorem for dynamics of continuous opinions. Physica A: Statistical Mechanics and its Applications, 335:217-223, 2005.

[13] L. Moreau. Stability of multiagent systems with time-dependent communication links. IEEE Trans. on Automatic Control, 50(2):169-182, 2005.

[14] I.-C. Morărescu and A. Girard. Opinion dynamics with decaying confidence: Application to community detection in graphs. IEEE Trans. on Automatic Control, 56(8): 1862 - 1873, 2011.

[15] I.-C. Morărescu, S.-I. Niculescu, and A.Girard. Consensus with constrained convergence rate and time-delays. In In Proc. IFAC Worksop on Time-Delay Systems, 2010.

[16] M. E. J. Newman. Modularity and community structure in networks. Proc. Natl. Acad. Sci. USA, 103:8577-8582, 2006.

[17] M. E. J. Newman and M. Girvan. Finding and evaluating community structure in networks. Phys. Rev. E, 69: 026113, 2004.

[18] R. Olfati-Saber, J. A. Fax, and R. M. Murray. Consensus and cooperation in networked multi-agent systems. Proceedings of the IEEE, 95(1):215-233, 2007.

[19] R. Olfati-Saber and R.M. Murray. Consensus problems in networks of agents with switching topology and time-delays. IEEE Transactions on Automatic Control, 49:1520-1533, 2004.

[20] A. Olshevsky and J.N. Tsitsiklis. Convergence speed in distributed consensus and averaging. SIAM Journal of Control and Optimization, 48(1):33-55, 2009.

[21] G.A. Pavlopoulos, M. Secrier, C.N. Moschopoulos, T.G. Soldatos, S. Kossida, J. Aerts, R. Schneider, and P. G. Bagos. Using graph theory to analyze biological networks. BioData Mining, 4(10):1-27, 2011.

[22] O. Ratmann, C. Wiuf, and J. W. Pinney. From evidence to inference: Probing the evolution of protein interaction networks. HFSP Journal, 3(5):290-306, 2009.

[23] W. Ren and R. W. Beard. Consensus seeking in multiagent systems under dynamically changing interaction topologies. IEEE Trans. on Automatic Control, 50(5):655-661, 2005.

[24] C. Reynolds. Boids (flocks, herds, and schools: a distributed behavioral model). Website: http://www.red3d.com/cwr/boids/, 2001. 
[25] B. Touri and A. Nedic. On approximations and ergodicity classes in random chains. IEEE Transactions on Automatic Control, 57(11):2718-2730, 2012.

[26] L. Xiao and S. Boyd. Fast linear iterations for distributed averaging. System and Control Letters, 53:65-78, 2004. 